\title{
Comparison of the Use of Different Modes of Mechanical Oral Hygiene in Prevention of Plaque and Gingivitis
}

Nanning A.M. Rosema, * Mark F. Timmerman, * Paula A. Versteeg, *

Wim H. van Palenstein Helderman, ${ }^{\dagger}$ Ubele Van der Velden, ${ }^{*}$ and G.A. Van der Weijden*

Background: The objective of this study was to evaluate the effect of an oscillating/rotating/pulsating powered toothbrush on plaque and gingivitis prevention over a 9-month period.

Methods: The study had an examiner-masked, randomized, three-group parallel design. A total of 122 subjects $\geq 18$ years of age in good general health and with at least five teeth per quadrant and no pockets $\geq 5 \mathrm{~mm}$ were included. A 3 -week preexperimental period of extensive oral home care, including rinses, was started to improve gingival health. Professional oral hygiene instruction with a manual brush was provided. At baseline, subjects were assigned to one of three regimens: twice daily brushing with a manual toothbrush, a manual toothbrush and the use of floss, or a powered toothbrush. Subjects were professionally instructed in their regimen and given a prophylaxis. Two weeks later, oral hygiene reinforcement was provided. Gingival bleeding, plaque, staining, and gingival abrasion were assessed during the preexperimental period and at baseline, 10 weeks, and 6 and 9 months.

Results: There was a significant reduction in plaque and gingivitis from the preexperimental period to baseline. At 10 weeks and 6 and 9 months, the level of plaque was statistically significantly lower with the powered toothbrush versus the other two regimens $(P \leq 0.002)$. At 10 weeks and 6 months, the level of bleeding in the powered toothbrush group was statistically significantly lower versus manual brushing alone $(P \leq 0.024)$.

Conclusions: The powered toothbrush maintained lower plaque levels for 9 months following the 3 -week treatment phase better than the manual toothbrush with or without floss. The powered toothbrush showed significant benefits in preventing gingival bleeding versus manual brushing alone. All regimens were safe for oral tissues. J Periodontol 2008;79: 1386-1394.

\section{KEY WORDS}

Dental floss; dental plaque; gingivitis; toothbrushing.

\footnotetext{
* Department of Periodontology, Academic Center for Dentistry Amsterdam, Amsterdam, The Netherlands.

$\dagger$ Department of Preventive and Restorative Dentistry, Radboud University Nijmegen Medical Center, Nijmegen, The Netherlands.
}

$\mathrm{T}$ The presence of high levels of plaque found in most people is largely responsible for the widespread prevalence of gingivitis, which is socially and clinically undesirable. Löe et al. ${ }^{1}$ established the importance of plaque in the etiology of gingival inflammation. They also demonstrated that the reinstatement of thorough tooth cleaning after a period of no cleaning resulted in the reestablishment of healthy gingivae. Powered toothbrushes are now generally regarded to be more efficacious than manual toothbrushes in removing plaque and maintaining or improving the gingival condition. ${ }^{2-4}$ Experience has shown that they are efficient and surprisingly appealing to patients. ${ }^{5}$ Because of these reasons, they have a definite place in the oral hygiene program.

Studies $^{6-13}$ over the past decade showed that certain powered toothbrushes (e.g., oscillating-rotating) are effective at plaque removal and reducing the signs of gingival inflammation. They are capable of effectively reestablishing gingival health after a period of experimentally induced inflammation. ${ }^{14-16}$

Although it is generally recognized that mechanical cleaning is potentially useful in controlling supragingival plaque, the expectation that each individual will maintain a good standard seems to be beyond most people's capabilities. Few people can sustain the dedication required to 
consistently perform this mechanical tooth-cleaning regimen.

The aim of the present study was to test whether a study population having moderate gingivitis was able to maintain reduced levels of plaque and an improved gingival situation, as obtained after a 3-week preexperimental treatment, with the aid of different modes of oral hygiene procedures over a period of 9 months.

\section{MATERIALS AND METHODS}

\section{Subjects}

A total of 122 subjects (non-dental students) were recruited after screening to take part in the study. The volunteers were informed of the study in a recruitment letter and at the first appointment. They were given a written explanation of the background of the study, its objectives, and their involvement. After being screened for their suitability, they all gave their written informed consent. Subjects were required to fulfill the following criteria: $\geq 18$ years of age, a minimum of five evaluable teeth in each quadrant (with no partial dentures, orthodontic banding, or wires), an absence of oral lesions and/or periodontal pockets $>5 \mathrm{~mm}$, a level of gingival bleeding $>40 \%$, and the absence of pregnancy, systemic diseases (such as diabetes), and any adverse medical history or long-term medication. In addition these subjects were non-users of dental floss.

The study was approved by the Medical Ethics Committee of the Academic Medical Center (AMC) of Amsterdam (approval \#: MEC 05/035 \#05.17.0679).

Subjects were randomly assigned to one of three groups: manual brushing with no interdental cleaning (MB), manual brushing and floss (MBF), or powered brushing and no interdental cleaning (PB) (Fig. 1).

\section{Products}

Powered brush. The powered toothbrush ${ }^{\ddagger}$ has an oscillating/rotating/pulsating motion with an oscillation angle of $45^{\circ}$. Subjects were instructed to use the brush in the preset high-speed modus. The brush head $\$$ refill has an elliptical head that is $17 \mathrm{~mm}$ long and $13.8 \mathrm{~mm}$ wide. Four tufts are free to pivot about their base, and 19 tufts are stapled.

Manual brush. The manual toothbrush ${ }^{\|}$has cylindrical filaments with end-rounded filament ends. The brush has 47 tufts and $\sim 40$ filaments per tuft. The straight handle is rectangular shaped and consists only of hard material.

Dental floss. Waxed satin floss ${ }^{\text {Il }}$ was used.

All products and a standard (NaF) dentifrice ${ }^{\#}$ were supplied throughout the study free of charge.

\section{Clinical Parameters}

Gingival condition was assessed using the bleeding on marginal probing (BOMP) index; ${ }^{17}$ the gingival margin was probed at $\sim 60^{\circ}$ to the longitudinal axis of

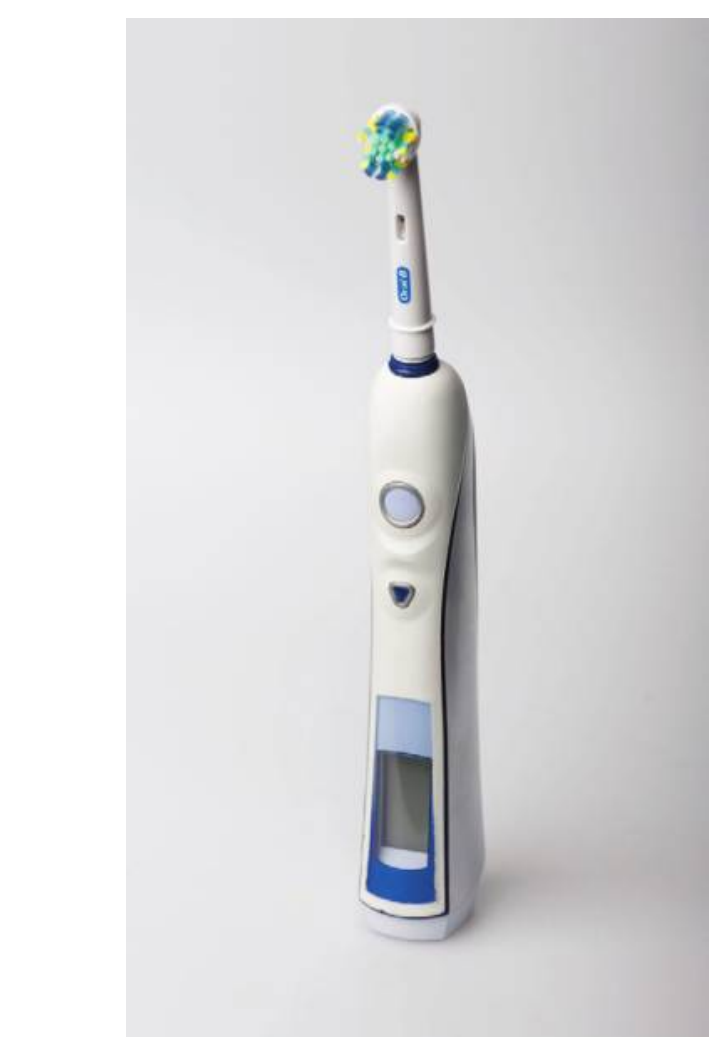

Figure I.

The powered toothbrush.

the tooth, and the absence or presence of bleeding was scored within 30 seconds of probing on a scale of 0 to 2 ( $0=$ non-bleeding; 1 = pinprick bleeding; 2 = excess bleeding). Six surfaces were examined per tooth (disto-buccal, mid-buccal, mesio-buccal, disto-lingual, mid-lingual, and mesio-lingual). Subsequently, staining of teeth at the vestibular sides was scored according to the Gründemann Modification of the Staining Index (GMSI), ${ }^{18}$ recording four areas per tooth (two approximal, one gingival, and one incisal). Staining at these areas was assessed according to the intensity stain index of Lobene. ${ }^{19}$

Next, plaque was disclosed using a new cotton swab with fresh disclosing solution** for each quadrant. Plaque was assessed using the modified Quigley and Hein plaque index (QHPI) as described in detail by Paraskevas et al. ${ }^{20}$ the absence or presence of plaque was recorded on a six-point scale $(0$ to $5 ; 0=$ no plaque, and 5 = plaque covered more than two-thirds

‡ Oral-B Triumph Professional Care 9000 (D25), Procter \& Gamble, Cincinnati, $\mathrm{OH}$.

$\S$ Oral-B Floss Action (EB25), Oral-B Laboratories, Procter \& Gamble.

\| ADA Soft reference toothbrush, American Dental Association, Chicago, IL.

I Oral-B Satin floss, Procter $\&$ Gamble.

\# Zendium frismint, RDA +/-76, Sara Lee HEBC, Veenendaal, The Netherlands.

** Mira-2-Ton, Hager \& Werken, Duisburg, Germany. 
of the tooth surface) at the same sites as for gingival bleeding.

Subsequently, gingival abrasion lesions were scored (GAS) according to the method described by Van der Weijden et al. ${ }^{21}$ and Versteeg et al. ${ }^{22}$ For visualization of the number and site of any gingival abrasions (third molar regions were excluded), the gums were also disclosed using disclosing solution. The gingival tissues were divided into three areas: marginal (cervical free gingiva), approximal (papillary free gingiva), and mid-gingival (attached gingiva). A periodontal probe ${ }^{\dagger \dagger}$ placed across the long axis of the lesions, was used to measure the size of the abrasions, and the greatest diameter of the lesion was recorded.

Clinical examinations were performed at day 0 , baseline, 10 weeks, 6 months, and 9 months. All parameters assessed at the start of the study were evaluated at each occasion, except for stain at baseline. All examinations were performed throughout the study by the same experienced examiners (PAV: GMSI and BOMP; NAMR: QHPI and GAS) under the same conditions. The examiners were masked to treatment randomization, and records of earlier examinations were not available at the time of reexaminations.

\section{Study Design}

The present study used a randomized, examinermasked, half-mouth, three-group parallel design consisting of two phases: a preexperimental phase and an experimental period with a total duration of 9 months (Fig. 2). At the start of the preexperimental phase (day 0), subjects were assessed for the clinical parameters BOMP, plaque, gingival abrasion, and stain using a partial-mouth scoring model. ${ }^{23}$ All teeth in two randomly chosen contralateral quadrants (one upper and one lower quadrant) were examined except for third molars. To obtain optimal periodontal health, subjects received thorough professional instruction in the use of a manual toothbrush with standard toothpaste. They were instructed to use these products for the next 3 weeks, according to the Bass technique, ${ }^{24}$ brushing twice daily for 2 minutes. A timer was provided to keep track of time. In addition, subjects were instructed to rinse twice a day with a hydrogen peroxide solution ${ }^{\ddagger}$ immediately followed by chlorhexidine (CHX) $0.2 \%$ mouthwash $\$ \S$ until the second phase of the experiment commenced. This procedure was shown to be effective in reducing plaque and gingival bleeding. ${ }^{18,25}$ Written instructions were given, and a calendar was provided to record brushing and rinsing times to check compliance. The purpose of this preexperimental phase was to motivate the subjects and to improve the level of gingival health. Subjects were instructed to brush between 2 and 3 hours before their appointment to avoid the risk of increased bleeding as a result of toothbrushing. ${ }^{26}$

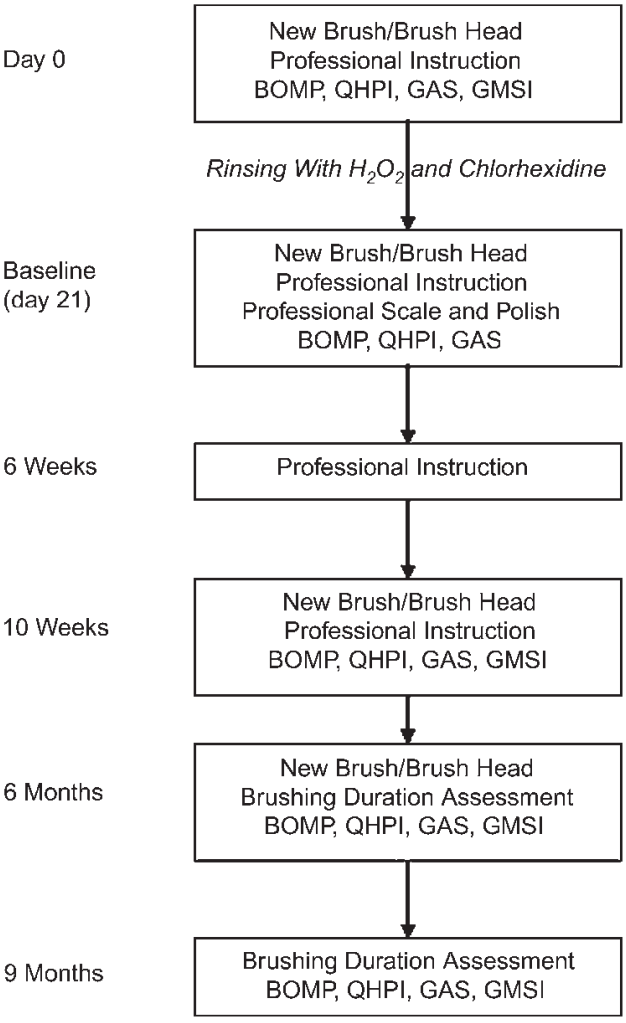

Figure 2. Flow chart.

The second appointment, for the baseline assessment (QHPI, BOMP, and GAS), was scheduled 3 weeks after the first appointment. After examination, a hygienist provided a professional dental scale and polish to enter subjects into the experimental phase of the study with equally clean teeth. At this time subjects were assigned to one of the three groups.

Randomization was performed using true random numbers that are generated by sampling and processing a source of entropy outside the computer.

All subjects received their assigned toothbrush and floss, if appropriate. Volunteers in the PB group were instructed according to the manufacturer's instructions. Subjects in MB and MBF groups received reinforcement in instruction according to the Bass technique. ${ }^{24}$ Participants in the MBF group received additional instruction regarding the use of floss. ${ }^{27}$

At the baseline, 10-week, and 6-month appointments, all subjects received a new brush head or toothbrush and floss, depending on group allocation, as well as a sufficient amount of dentifrice to last until the next appointment. Old brush heads and toothbrushes were collected when providing new ones

$\dagger \dagger \mathrm{PQ}$-Williams, Hu-Friedy, Chicago, IL.

¥ Bocasan, Oral-B Laboratories, Procter $\mathcal{E}$ Gamble, Mason, $\mathrm{OH}$.

$\S \S$ Corsodyl, GlaxoSmithKline, Zeist, The Netherlands. 
to ensure that all subjects started the next period with fresh products.

All subjects returned at 6 weeks for a second oral hygiene instruction. The assigned brushing technique was reinforced in all three groups. Also, the use of floss was reinforced, depending on the assigned regimen. Subjects returned 4 weeks later for their 10-week examination, and they received their last oral hygiene instruction and reinforcement. No additional instructions were given during the remainder of the study. At the 6- and 9-month visits, all subjects were asked to brush their teeth as they would do at home following the clinical assessments. Meanwhile, brushing time was recorded using a stopwatch, an action of which subjects were unaware.

\section{Data Analyses}

The individual measurements were summarized within each individual and then analyzed. Means for BOMP, QHPI, and GAS were calculated. GMSI scores were calculated based on percentages of examined sites. Comparisons between regimens were made for QHPI and BOMP using a three-level repeated-measures analysis with measurements at 10 weeks, 6 months, and 9 months as dependent variables and day 0 and baseline scores as covariates. Residual analyses confirmed validity of the calculated $P$ values. For explorative analysis, the incremental changes between the baseline and the 10-week, 6-month, and 9-month examinations were calculated, and non-

\section{Table I.}

\section{Demographic Data of the Study Population}

\begin{tabular}{lccc}
\hline Characteristic & MB Group & MBF Group & PB Group \\
\hline $\mathrm{n}$ & 38 & 39 & 37 \\
Age (years; mean \pm SD) & $21.6 \pm 2.54$ & $22.2 \pm 3.25$ & $22.4 \pm 2.93$ \\
Female/male $(n)$ & $32 / 6$ & $32 / 7$ & $28 / 9$ \\
Smokers $(n)$ & 5 & 5 & 2 \\
\hline
\end{tabular}

parametric tests were used. Unadjusted $P$ values were reported for the proper interpretation of the effects and the influence of overall tests as applied to primary response variables. For GAS, overall scores were tested using the analysis of variance (ANOVA) test to compare scores among regimens at each assessment. For BOMP, QHPI and GMSI, overall scores were tested using the Kruskal-Wallis test to compare scores among regimens at each assessment. For brushing time, mean scores were tested using the Mann-Whitney test to compare scores among regimens at both assessments. Pvalues $<0.05$ were considered statistically significant.

\section{RESULTS}

\section{Study Population (Table 1)}

A total of 122 subjects were recruited, of whom 114 completed the 9-month protocol between March and December 2005 at Academic Centre for Dentistry Amsterdam. Four subjects did not show up for their first appointment. A total of 118 subjects were divided among the three groups. Two subjects (one in the MBF group and one in the PB group) failed to attend the baseline visit because of scheduling conflicts. Two subjects were lost at the 9-month visit; one subject (MB group) was hospitalized as a result of a leg injury, and one had moved to a different part of the country. All subjects were in good general health and were not taking any medication that interfered with the study outcomes. Based on returned calendars supplied at the start of the preexperimental phase, compliance for the twice-daily brushing regimen followed by the use of the two mouthwashes was nearly $100 \%$.

\section{Bleeding (Table 2)}

Bleeding scores decreased significantly for all groups $(P<0.001)$ during the preexperimental phase (day 0 to baseline). Although a significant difference among the three groups was present at day 0 , no significant differences could be detected among them at baseline. In this period, groups had not been assigned to their specific oral hygiene procedure, and all subjects

Table 2.

Bleeding Scores (BOMP; mean \pm SD)

\begin{tabular}{lcccccc}
\hline & $\mathrm{n}$ & Day 0 & Baseline & 10 Weeks & 6 Months & 9 Months \\
\hline MB group & 38 & $1.25 \pm 0.34$ & $0.57 \pm 0.26$ & $0.47 \pm 0.30$ & $0.59 \pm 0.31$ & $0.65 \pm 0.30$ \\
MBF group & 39 & $0.98 \pm 0.38$ & $0.49 \pm 0.26$ & $0.38 \pm 0.22$ & $0.40 \pm 0.19$ & $0.58 \pm 0.27$ \\
PB group & 37 & $1.07 \pm 0.39$ & $0.57 \pm 0.27$ & $0.32 \pm 0.20$ & $0.39 \pm 0.27$ & $0.57 \pm 0.36$ \\
$P$ value* & & 0.007 & 0.246 & 0.024 & 0.002 & 0.221 \\
\hline
\end{tabular}

* Kruskal-Wallis test. 
Table 3.

Plaque Scores (QHPI; mean \pm SD)

\begin{tabular}{lcccccc}
\hline & $n$ & Day 0 & Baseline & 10 Weeks & 6 Months & 9 Months \\
\hline MB group & 38 & $2.18 \pm 0.49$ & $1.05 \pm 0.57$ & $1.61 \pm 0.52$ & $1.59 \pm 0.42$ & $1.57 \pm 0.57$ \\
MBF group & 39 & $1.95 \pm 0.39$ & $0.71 \pm 0.30$ & $1.61 \pm 0.42$ & $1.52 \pm 0.38$ & $1.44 \pm 0.45$ \\
PB group & 37 & $1.97 \pm 0.42$ & $0.86 \pm 0.40$ & $1.21 \pm 0.50$ & $1.21 \pm 0.41$ & $1.16 \pm 0.47$ \\
$P$ value* & & 0.047 & 0.032 & 0.002 & $<0.001$ & 0.002 \\
\hline
\end{tabular}

* Kruskal-Wallis test.

used both mouthwashes twice daily. The overall repeated-measures analysis showed statistically significant differences among all three groups $(P=0.027)$ with day 0 and baseline entered as covariates. Posttesting showed a statistically significant difference only between MB and PB groups $(P=0.026)$.

At the 10-week visit, bleeding scores were the lowest of the study. At this point, the MB group had an overall bleeding score of 0.47 . This score was 0.38 for the MBF group and 0.32 for the PB group. The difference among groups was statistically significant $(P=$ $0.024)$. At the 6 -month visit, the bleeding scores were higher for all three regimens. The differences among the three groups were statistically significant $(P=$ 0.002). At the final visit, the overall bleeding scores had increased again for all regimens. The MB group had a score of 0.65 , which was slightly higher compared to the 6-month visit. MBF and PB groups showed similar changes, with scores of 0.58 and 0.57 , respectively. No significant difference among groups could be detected at this point.

\section{Plaque (Table 3)}

During the preexperimental phase (day 0 to baseline), plaque scores decreased significantly $(P<0.001)$ for all groups, although the $M B$ group started at day 0 with a significantly higher score compared to the other two groups. A significant difference $(P=0.032)$ among the three groups could still be detected at baseline. Overall plaque scores were 1.05 for the $M B$ group, 0.71 for the MBF group, and 0.86 for the PB group. For all three groups this was the lowest overall plaque score of the study. The overall repeated-measures analysis showed statistically significant differences among all three groups $(P<0.001)$ with day 0 and baseline entered as covariates. Post-testing showed statistically significant differences between the PB group and both the MB and MBF groups $(P \leq 0.001)$.

At the 10-week visit, higher plaque scores were observed for all regimens. Statistically significant differences were observed among the three groups $(P=$ $0.002)$. At the 6 -month visit, the plaque scores of $M B$ and PB groups were similar to those observed at the
Table 4.

\section{5\% Confidence Intervals for BOMP and QHPI}

\begin{tabular}{lrrrr}
\hline & 10 Weeks & 6 Months & 9 Months \\
\hline BOMP & & & \\
PB versus MB & 0.04 to 0.27 & 0.07 to 0.34 & -0.07 to 0.24 \\
PB versus MBF & -0.04 to 0.18 & -0.09 to 0.12 & -0.13 to 0.16 \\
QHPI & & & \\
PB versus MB & 0.16 to 0.63 & 0.19 to 0.58 & 0.17 to 0.65 \\
PB versus MBF & 0.19 to 0.61 & 0.13 to 0.49 & 0.07 to 0.49 \\
\hline
\end{tabular}

10-week visit; however, the score for the MBF group was slightly lower compared to the previous visit. Similar to the 10 -week visit, the PB group had a statistically significant $(P<0.001)$ lower overall plaque score compared to the other two groups. At the final visit at 9 months, the overall plaque scores were lower for all regimens compared to the 6-month assessment. The MB group had a score of 1.57 , and MBF and PB groups had scores of 1.44 and 1.16, respectively. Again, significant differences between the PB group and the other two groups were detected $(P=$ 0.002). Table 4 shows the $95 \%$ confidence intervals.

\section{Gingival Abrasion (Table 5)}

The overall GAS for all groups were comparable at day 0 . Lower GAS were observed at the baseline visit, but no significant differences among the three regimens were detected. At the 10 -week visit, the overall GAS reached levels similar to those at day 0 . Comparable scores were observed at the 6 -month visit, and the highest scores of the study were noted at the 9 -month visit. Differences among groups did not reach statistical significance throughout the study.

\section{Staining (Table 6)}

The overall staining scores for all groups were comparable at day 0 . Staining was not assessed at the baseline visit because all subjects rinsed with both mouthwashes, resulting in possible staining caused 
Table 5.

Gingival Abrasion Scores (GAS; mean \pm SD)

\begin{tabular}{lcccccc}
\hline & $\mathrm{n}$ & Day 0 & Baseline & 10 Weeks & 6 Months & 9 Months \\
\hline MB group & 38 & $4.34 \pm 3.29$ & $2.21 \pm 2.06$ & $4.61 \pm 5.48$ & $4.21 \pm 3.38$ & $7.82 \pm 6.90$ \\
MBF group & 39 & $4.82 \pm 3.97$ & $2.95 \pm 2.75$ & $4.31 \pm 3.45$ & $4.26 \pm 3.39$ & $6.03 \pm 3.98$ \\
PB group & 37 & $5.11 \pm 3.70$ & $2.43 \pm 2.08$ & $4.24 \pm 3.04$ & $4.54 \pm 4.38$ & $6.70 \pm 6.14$ \\
P value* & & 0.620 & 0.580 & 0.719 & 0.994 & 0.580 \\
\hline
\end{tabular}

* ANOVA test.

Table 6.

Staining Scores (GMSI; percentages of sites \pm SD)

\begin{tabular}{|c|c|c|c|c|c|c|}
\hline & $n$ & Day 0 & Baseline & 10 Weeks & 6 Months & 9 Months \\
\hline MB group & 38 & $4.00 \pm 5.63$ & NA & $5.74 \pm 7.43$ & $5.17 \pm 7.06$ & $7.51 \pm 6.84$ \\
\hline MBF group & 39 & $4.45 \pm 5.44$ & NA & $3.95 \pm 4.72$ & $3.73 \pm 4.35$ & $6.17 \pm 4.80$ \\
\hline PB group & 37 & $2.96 \pm 4.96$ & NA & $3.91 \pm 5.97$ & $3.53 \pm 5.48$ & $4.74 \pm 5.37$ \\
\hline$P$ value* & & 0.4616 & NA & 0.2529 & 0.5368 & 0.1004 \\
\hline
\end{tabular}

NA = not applicable

* Kruskal-Wallis test.

by CHX. At this point in the study, all subjects received a professional debridement and polish to remove all staining. At the 3-month visit, the overall staining scores (percentage of sites) ranged from 3.91 for the PB group to 5.74 for the MB group. At the 6 -month visit, all groups showed similar values, and the scores showed a slight increase at the 9-month visit. However, the differences among groups did not reach statistical significance throughout the study.

\section{Brushing Time}

The brushing time was measured at the 6- and 9 -month visits, and means were calculated for each group. The recorded brushing time at 6 months was 129.5 seconds for the PB group, 121.6 seconds for the MBF group, and 111.3 seconds for the MB group. This resulted in a statistically significant difference between the PB and MB groups $(P=0.006)$. At 9 months, the brushing times were 119.4 seconds (PB), 120.3 seconds (MBF), and 116.5 seconds (MB).

\section{DISCUSSION}

Since their introduction in the early 1960 s, powered toothbrushes have been challenged with the task of providing a better cleaning option than manual brushes. Research has demonstrated that the plaqueremoving efficacy of electric brushes has improved, and powered toothbrushes are considered viable alternatives to manual brushing by professionals and patients. ${ }^{4}$ The daily use of a toothbrush is known to be essential to maintain gingival health. However, people often fail to maintain optimal gingival health over a long period of time.

The design of the present study was based on a model published by Svatun et al. ${ }^{28,29}$ and tested the concept that good gingival health can be maintained with the use of a prophylactic aid. Without the use of such an aid, improved gingival health tends to fade over time and returns to its original values. The most marked deterioration occurs within the first 3 months following the preexperimental phase, indicating a relatively rapid loss of the dedication that is required to maintain a high degree of plaque control. ${ }^{30}$ In both Svatun et al. ${ }^{28,29}$ studies, the moderately inflamed gingival condition of a group of young, healthconscious volunteers was brought to an excellent state of health by professional cleaning and oral hygiene instruction. This study model proved to be effective in testing oral hygiene aids to suppress plaque accumulation and the development of gingivitis.

The model of Svatun et al. ${ }^{28}$ was adapted for the present study. During the 3-week preexperimental phase, the oral hygiene instruction was combined with the use of $\mathrm{H}_{2} \mathrm{O}_{2}$ and $\mathrm{CHX}$ rinses. The use of both mouthrinses was added to enable subjects to enter the experimental phase with the healthiest possible gingival condition. This was considered to provide the opportunity to discern maximum differences in gingivitis levels between day 0 and baseline. 
Previous research on toothbrushes has mainly focused on the reversal of gingivitis or the effect of various modes of oral hygiene on inflammation. ${ }^{14,16,22}$ However, limited data are available for a healthy study population that has the potential to develop gingivitis based on their intake gingival level. The results of the present study showed that the electric brush was significantly more effective in maintaining low plaque levels compared to the manual brush with or without the daily use of dental floss during the 9 months of the trial. With respect to the gingival condition, the powered brush, as well as the manual brush with the daily use of dental floss, was significantly more effective in maintaining low bleeding scores compared to the manual brush without the use of dental floss for a period of 6 months. At the 9-month assessment, this difference in gingival condition was no longer significant, although the plaque scores for the PB group at this visit were the lowest of the experimental period. An explanation for this could be that plaque scores reflect the current oral hygiene performance, whereas bleeding scores reflect the oral hygiene performance over a longer period. ${ }^{31}$ This is a clear indication for the need of reinforcement and professional instruction in the use of oral hygiene aids at $\sim 6$-month intervals, the typical recall interval, to obtain the maximum benefit from oral hygiene products.

Brushing duration is also an important factor. ${ }^{32-34}$ Average brushing times $<60$ seconds were reported in several studies. ${ }^{35-37}$ However, when pursuing maximum results from brushing, a duration $\geq 2$ minutes is advised. ${ }^{38,39}$ The results of the brushing-time assessments made at the 6- and 9-month visits showed that subjects were compliant with the instructions. This may have contributed to the decreasing plaque and bleeding scores in all three groups. Because all subjects were provided with a timer to keep track of brushing time, it was interesting to find a significant difference in duration between MB and PB groups at 6 months. Although this difference was not maintained at the 9-month visit, one could suggest that subjects brushing with a powered toothbrush with an incorporated 2-minute timer tended to brush longer than those using a manual brush.

Compared to the powered toothbrush group, it was hypothesized that the manual toothbrush group would not maintain low levels of plaque. In the study by Svatun et al., ${ }^{28}$ a chemically active dentifrice was tested, which implied that it was not necessary to repeat oral hygiene instruction as provided at the pretrial assessment. This was clearly different than in the present study in which different modes of mechanical oral hygiene were evaluated. These products require a professional instruction and training to achieve the maximum cleaning performance. ${ }^{40,41}$ Therefore, reinforcements of instructions were given at baseline and at 6 and 10 weeks. Subjects received thorough professional instructions at the clinic, and written instructions regarding the use of the assigned products to take home; it seems likely that this influenced the original model. In retrospect, it might be considered an important aspect of the present study that there were still interventions of oral hygiene instructions after the preexperimental period. These instructions could have contributed to the fact that, in contrast to the study by Svatun et al., the control group did not return to baseline levels. After 10 weeks and 6 and 9 months, subjects in all three groups still showed statistically significantly lower plaque and bleeding scores compared to day 0. Despite this, statistically significant differences in plaque scores among the three groups persisted from the 3-month visit. However, clinically significant improvement is not a statistical issue; it is decided based on clinical arguments. It is up to the reader to decide whether he or she considers the magnitude of improvement to be of clinical relevance; $95 \%$ confidence intervals are presented to help in this decision (Table 4).

Data from the present study showed that all groups had significantly lower mean bleeding and plaque scores at baseline after the preexperimental phase compared to day $0(P<0.001)$. Subjects used $\mathrm{H}_{2} \mathrm{O}_{2}$ and $\mathrm{CHX}$ in addition to toothbrushing during this preexperimental phase. Positive results, with respect to plaque inhibition, were reported when $\mathrm{CHX}$ was combined with oxidizing agents. ${ }^{18,24,42-47}$ Based on these findings, this regimen was incorporated into the present study to establish maximum improvement in gingival condition. Data from the present study showed that, together with a single oral hygiene instruction, the combined use of $\mathrm{H}_{2} \mathrm{O}_{2}$ and $\mathrm{CHX}$ in a twice-daily regimen proved to be effective in reducing plaque levels $>50 \%$ and bleeding levels $\sim 50 \%$ in a group of subjects with moderate gingivitis.

Gingival abrasion is a known side effect of toothbrushing. Excessive brushing force can traumatize oral soft and hard tissues. ${ }^{48}$ No adverse effects were reported in the present study, and there were no statistically significant differences in GAS scores among groups. The data showed that all regimens were safe. Another study ${ }^{49}$ testing electric and/or manual brushes and assessing gingival abrasion came to similar conclusions. With respect to GMSI, all subjects started the experimental phase after receiving a professional polish to remove deposits, such as stains. No statistically significant difference among groups was observed from day 0 throughout the experimental phase.

\section{CONCLUSIONS}

All regimens maintained lower levels of plaque and bleeding compared to the intake levels at day 0 . However, the powered toothbrush maintained lower 
plaque levels for 9 months more effectively and maintained an improved gingival condition for $\geq 6$ months compared to the manual toothbrush with or without the use of floss.

\section{ACKNOWLEDGMENTS}

The authors thank MaryAnn Cugini, senior clinical investigator, director of External Research Collaborations, The Forsyth Institute, and Paul Warren, vice president, Global Professional and Scientific Relations, PEG Professional Oral Health, for their input into the study design and Lisa Sagel, associate manager, Scientific Communications, PEG Professional Oral Health, for her assistance with the preparation of this manuscript. The authors thank Sara Lee DE International, Veenendaal, The Netherlands, for providing the dentifrice, and GlaxoSmithKline, Zeist, The Netherlands, for providing CHX. This study was sponsored by Procter \& Gamble, Cincinnati, Ohio. N.A.M. Rosema has received lecture fees from Procter $\mathcal{G}$ Gamble, and G.A. Van der Weijden has received advising fees from Procter \& Gamble. M.F. Timmerman, P.A. Versteeg, W.H. van Palenstein Helderman, and U. Van der Velden report no conflicts of interest related to this study.

\section{REFERENCES}

1. Löe H, Theilade E, Jensen SB. Experimental gingivitis in man. J Periodontol 1965;36:177-187.

2. Warren PR, Chater B. The role of the electric toothbrush in the control of plaque and gingivitis: A review of 5 years clinical experience with the Braun Oral-B Plaque Remover [D7]. Am J Dent 1996;9:S5S11.

3. Saxer UP, Yankell SL. Impact of improved toothbrushes on dental diseases. II. Quintessence Int 1997;28:573593.

4. Warren PR. Development of an oscillating/rotating/ pulsating toothbrush: The Oral-B ProfessionalCare Series. J Dent 2005;33(Suppl. 1):1-9.

5. Walters PA, Cugini M, Biesbrock AR, Warren PR. A novel oscillating-rotating power toothbrush with SmartGuide: Designed for enhanced performance and compliance. J Contemp Dent Pract 2007;8:1-9.

6. Tritten CB, Armitage GC. Comparison of a sonic and a manual toothbrush for efficacy in supragingival plaque removal and reduction of gingivitis. J Clin Periodontol 1996;23:641-648.

7. Baab DA, Johnson RH. The effect of a new electric toothbrush on supragingival plaque and gingivitis. J Periodontol 1989;60:336-341.

8. Johnson BD, McInnes C. Clinical evaluation of the efficacy and safety of a new sonic toothbrush. J Periodontol 1994;65:692-697.

9. Schifter CC, Emling RC, Seibert JS, Yankell SL. A comparison of plaque removal effectiveness of an electric versus a manual toothbrush. Clin Prev Dent 1983;5: 15-19.

10. Forrest JL, Miller SA. Manual versus powered toothbrushes: A summary of the Cochrane Oral Health Group's Systematic Review. Part II. J Dent Hyg 2004; 78:349-354.
11. Sicilia A, Arregui I, Gallego M, Cabezas B, Cuesta S. A systematic review of powered vs manual toothbrushes in periodontal cause-related therapy. J Clin Periodontol 2002;29(Suppl. 3):39-54.

12. Heanue M, Deacon SA, Deery C, et al. Manual versus powered toothbrushing for oral health. Cochrane Database Syst Rev 2003;1:CD002281.

13. Robinson PG, Deacon SA, Deery C, et al. Manual versus powered toothbrushing for oral health. Cochrane Database Syst Rev 2005;2:CD002281.

14. van der Weijden FA, Timmerman MF, Piscaer $M$, IJzerman Y, Warren PR, van der Velden U. A comparison of the efficacy of a novel electric toothbrush and a manual toothbrush in the treatment of gingivitis. $\underline{A m ~ J}$ Dent 1998;11:S23-S28.

15. van der Weijden GA, Timmerman MF, Piscaer $M$, IJzerman Y, van der Velden U. A clinical comparison of three powered toothbrushes. $J$ Clin Periodontol 2002;29:1042-1047.

16. Rosema NAM, Timmerman MF, Piscaer M, et al. An oscillating/pulsating electric toothbrush versus a highfrequency electric toothbrush in the treatment of gingivitis. J Dent 2005;33(Suppl. 1):29-36.

17. Lie MA, Timmerman MF, van der Velden $U$, van der Weijden GA. Evaluation of 2 methods to assess gingival bleeding in smokers and non-smokers in natural and experimental gingivitis. J Clin Periodontol 1998; 25:695-700.

18. Gründemann LJ, Timmerman MF, Ijzerman Y, van der Velden U, van der Weijden GA. Stain, plaque and gingivitis reduction by combining chlorhexidine and peroxyborate. J Clin Periodontol 2000;27:9-15.

19. Lobene RR. Effect of dentifrices on tooth stains with controlled brushing. J Am Dent Assoc 1968;77:849855.

20. Paraskevas S, Rosema NAM, Versteeg P, Timmerman $M F$, van der Velden $U$, van der Weijden GA. The additional effect of a dentifrice on the instant efficacy of toothbrushing: A crossover study. JPeriodontol 2007; 78:1011-1016.

21. van der Weijden GA, Timmerman MF, Versteeg PA, Piscaer $M$, van der Velden U. High and low brushing force in relation to efficacy and gingival abrasion. $\underline{J \text { Clin }}$ Periodontol 2004;8:620-624.

22. Versteeg PA, Timmerman MF, Rosema NAM, Warren PR, van der Velden U, van der Weijden GA. Sonicpowered toothbrushes and reversal of experimental gingivitis. J Clin Periodontol 2005;32:1236-1241.

23. Bentley CD, Disney JA. A comparison of partial and full mouth scoring of plaque and gingivitis in oral hygiene studies. J Clin Periodontol 1995;22:131-135.

24. Bass CC. An effective method of personal oral hygiene. J La State Med Soc 1954;106:100-112.

25. Dona BL, Gründemann LJ, Steinfort J, Timmerman $M F$, van der Weijden GA. The inhibitory effect of combining chlorhexidine and hydrogen peroxide on 3-day plaque accumulation. J Clin Periodontol 1998; 25:879-883.

26. Abbas F, Voss S, Nijboer A, Hart AA, van der Velden $U$. The effect of mechanical oral hygiene procedures on bleeding on probing. J Clin Periodontol 1990;17: 199-203.

27. Wilkins EM. Clinical Practice of the Dental Hygienist, 8th ed. London: Lippincott Williams E Wilkins;1999: 373-375.

28. Svatun B, Saxton CA, van der Ouderaa F, Rölla G. The influence of a dentifrice containing a zinc salt and a 
nonionic antimicrobial agent on the maintenance of gingival health. J Clin Periodontol 1987;14:457-461.

29. Svatun B, Saxton CA, Rölla G, van der Ouderaa F. A 1 -year study on the maintenance of gingival health by a dentifrice containing a zinc salt and non-anionic antimicrobial agent. J Clin Periodontol 1989;16:75-80.

30. Saxton CA. A combination of therapeutic agents for the control of dental plaque and gingivitis in man. [Thesis]. Nijmegen, The Netherlands: Catholic University Nijmegen; 1993. 126 p.

31. Lang NP, Cumming BR, Löe H. Toothbrushing frequency as it relates to plaque development and gingival health. J Periodontol 1973;44:396-405.

32. van der Weijden GA, Timmerman MF, Piscaer $M$, Ijzerman Y, van der Velden U. Plaque removal by professional electric toothbrushing compared with professional polishing. J Clin Periodontol 2004;31: 903-907.

33. van der Weijden GA, Timmerman MF, Danser MM, Piscaer M, Ijzerman Y, van der Velden U. Approximal brush head used on a powered toothbrush. J Clin Periodontol 2005;32:317-322.

34. Williams K, Ferrante A, Dockter K, Haun J, Biesbrock AR, Bartizek RD. One- and 3-minute plaque removal by a battery-powered versus a manual toothbrush. J Periodontol 2004;75:1107-1113.

35. Macgregor ID, Rugg-Gunn AJ. Toothbrushing duration in 60 uninstructed young adults. Community Dent Oral Epidemiol 1985;13:121-122.

36. Macgregor ID, Rugg-Gunn AJ. Effect of filming on toothbrushing performance in uninstructed adults in north-east England. Community Dent Oral Epidemiol 1986; 14:320-322.

37. Beals D, Ngo T, Feng Y, Cook D, Grau DG, Weber DA. Development and laboratory evaluation of a new toothbrush with a novel brush head design. Am J Dent 2000;13:5A-14A.

38. van der Weijden GA, Timmerman MF, Nijboer A, Lie MA, van der Velden U. A comparative study of electric toothbrushes for the effectiveness of plaque removal in relation to toothbrushing duration. Timerstudy. J Clin Periodontol 1993;20:476-481.

39. van der Weijden FA, Timmerman MF, Snoek IM, Reijerse E, van der Velden U. Toothbrushing duration and plaque removing efficacy of electric toothbrushes. Am J Dent 1996;9:S31-S36.
40. van der Weijden GA, Timmerman MF, Reijerse E, et al. The long-term effect of an oscillating/rotating electric toothbrush on gingivitis. An 8-month clinical study. J Clin Periodontol 1994;21:139-145.

41. Jepsen S. The role of manual toothbrushes in effective plaque control: Advantages and limitations. In: Lang NP, Attström R, Löe H, eds. Proceedings of the European Workshop on Mechanical Plaque Control. Berlin: Quintessenz Verlag; 1998:121-137.

42. Solheim H, Eriksen HM, Nordb $\varnothing$ H. Chemical plaque control and extrinsic discoloration of teeth. Acta Odontol Scand 1980;38:303-309.

43. Eriksen HM, Solheim H, Nordb $\varnothing \mathrm{H}$. Chemical plaque control and prevention of extrinsic tooth discoloration in vivo. Acta Odontol Scand 1983;41:87-91.

44. Addy M, al-Arrayed F, Moran J. The use of an oxidising mouthwash to reduce staining associated with chlorhexidine. Studies in vitro and in vivo. J Clin Periodontol 1991;18:267-271.

45. Charbonneau DL, Snider AG. Reduced chlorhexidine tooth stain coverage by sequential administration of monoperoxyphthalic acid in the beagle dog. $\underline{J \text { Dent }}$ Res 1997;76:1596-1601.

46. Steinberg D, Heling I, Daniel I, Ginsburg I. Antibacterial synergistic effect of chlorhexidine and hydrogen peroxide against Streptococcus sobrinus, Streptococcus faecalis and Staphylococcus aureus. J Oral Rehabil 1999;26:151-156.

47. Winer RA, Chauncey HH, Garcia RI. Effect of Peroxyl mouthrinse on chlorhexidine staining of teeth. $\underline{J \text { Clin }}$ Dent 1991;3:15-18.

48. Alexander JF, Saffir AJ, Gold W. The measurement of the effect of toothbrushes on soft tissue abrasion. J Dent Res 1977;56:722-727.

49. van der Weijden GA, Timmerman MF, Piscaer $M$. Ijzerman Y, van der Velden U. Oscillating/rotating electric toothbrushes compared: Plaque removal and gingival abrasion. J Clin Periodontol 2001;28:536-543.

Correspondence: Dr. N.A.M. Rosema, Department of Periodontology, Academic Center for Dentistry Amsterdam, Louwesweg 1, 1066 EA Amsterdam, The Netherlands. E-mail: n.rosema@acta.nl.

Submitted December 18, 2007; accepted for publication February 5, 2008. 